\title{
The ISFET based heparin sensor with a monolayer of protamine as affinity ligand
}

\author{
J.C. van Kerkhof \& P. Bergueld \\ MESA Research Institute, University of Twente, P.O. Box 217, 7500 AE Enschede, The Netherlands \\ Tel: [31] 53892688 Fax: [31] 53328439
}

\section{R.B.M. Schasfoort}

TNO Nutrition and Food Research, P.O. Box 360, 3700 AJ Zeist, The Netherlands

(Received 5 May 1994; revised 8 August 1994; accepted 16 August 1994)

\begin{abstract}
The ion-step measuring method was used to determine absolute heparin concentrations in PBS and blood plasma with a $\mathrm{Ta}_{2} \mathrm{O}_{5}$ ISFET on to which a monolayer of protamine had been immobilized. Heparin is a highly negatively charged polysaccharide, which is used clinically to delay the clotting of blood. Protamine acts as an affinity ligand for heparin. The response of the ISFET system on a step-wise increase in the electrolyte concentration (a so-called ion-step) is a transient change of the output voltage, which is related to the surface charge density of the ISFET gate oxide. After 2 mins of incubation in a plasma sample containing heparin, the amplitude of the transient ISFET response to an ion-step showed a linear relation to the heparin concentration. In blood plasma, heparin concentrations between 0.3 and 2.0 Units $/ \mathrm{ml}$ could be determined with an accuracy of \pm 0.08 Units $/ \mathrm{ml}$. Heparin concentrations in different plasma samples of heparinized patients were determined and compared with the APTT. No direct relation was found between the APTT and the heparin concentration, but this result was not surprising.
\end{abstract}

Keywords: biosensor, ISFET, heparin, protamine

\section{INTRODUCTION}

In one of our previous papers we introduced an Ion-Selective Field Effect Transistor (ISFET) based heparin sensor using the ion-step measuring method (Van Kerkhof et al., 1993). On top of the ISFET was mounted a porous membrane, consisting of polystyrene beads in an agarose gel, in which the affinity ligand was immobilized.
Although it was possible to determine heparin concentrations in buffered solutions with this sensor, the results in blood plasma were disappointing due to a large interfering effect caused by other plasma components. In this paper we introduce a new approach to this type of heparin sensor. The affinity ligand, protamine, is in this case directly immobilized at the surface of the ISFET; this improves the response time of the 
sensor and decreases the effect of the other plasma components. With this sensor it is possible to determine heparin concentrations in blood plasma in the range of physiological importance.

\section{Heparin}

Heparin is a highly sulphated glycosaminoglycan (GAG), found in the liver, lung and other tissues, that has several pharmacological properties (Nader \& Dietrich, 1989). The property of heparin that has attracted most attention, and resulted in its widespread use, is its ability to prolong the clotting time of blood by preventing the formation of insoluble fibrin. The anticoagulant activity of heparin is used in vascular surgery and in the treatment of postoperative thrombosis and embolism.

Heparin is defined as a family of polysaccharide species, whose chains are made up of alternating 1,4-linked and variously sulphated residues of a uronic acid and D-glucosamine. The uronic acid residues are either $\mathrm{L}$-iduronic acid or $\mathrm{D}$ glucuronic acid, and the D-glucosamine residues are either $\mathrm{N}$-sulphated or $\mathrm{N}$-acetylated (Casu, 1989). The molecular weight of the heparin fragment ranges +5 between 5000 and 30000 , averaging between 12000 and 15000 . The polysaccharide chains are structurally heterogeneous because of an incomplete biosynthetic modification of the precursors of heparin. At physiological $\mathrm{pH}$ values the three acidic functional groups in heparin are fully dissociated to yield $-\mathrm{OSO}_{3}^{-}$, $-\mathrm{NHSO}_{3}^{-}$, and $-\mathrm{COO}^{-}$groups, which give rise to a highly negatively charged heparin molecule.

In the blood coagulation process, more than ten different proteins are involved, which are called coagulation (or clotting) factors and which are labelled with roman numerals. Most of these proteins are (pro-)enzymes that activate each other in a cascade reaction. The result of this chain reaction is the formation of the enzyme thrombin, which plays a crucial role in the clotting process. Thrombin converts the soluble protein fibrinogen into insoluble fibrin, which forms the basis of a blood clot. To control the blood coagulation process, there are many positive and negative feedback mechanisms within the coagulation process itself, and between the coagulation process and other processes. One of the major ways in which coagulation is controlled is by the existence of inhibitory proteins, components of normal blood. The anticoagulant activity of heparin is based on its ability to increase the inhibitory effect of some of these inhibitors. Antithrombin III (AtIII) is considered to be the most important inhibitor of the coagulation process and not only inhibits thrombin, as the name indicates, but also other coagulation factors. However, thrombin and factor $\mathrm{Xa}$ are most sensitive to inactivation. Heparin binds to AtIII and produces a conformational change, thereby converting AtIII from a progressive, slow inhibitor to a very rapid inhibitor. After binding of this AtIII-heparin complex to a thrombin or factor Xa molecule, heparin dissociates from the complex and is reusable (Rosenberg, 1987; Björk et al., 1989). Only about one third of the molecules in normal heparin preparations are able to bind to AtIII and induce anticoagulant activity (Höök et al., 1976). The molecules that bind to AtIII each contain a unique pentasaccharide unit, which was found to be the binding site for AtIII (Casu, 1989).

Heparin is one of a variety of drugs extracted from natural sources, whose activity can not be adequately described or predicted by chemical and physical methods. In spite of the enormous advances made in recent years concerning knowledge of the chemical basis for heparin's anticoagulant activity (Casu, 1989; Petitou, 1989), the assessment of heparin for quality control purposes by manufacturers and for dosage by clinicians is still made in units of biological activity. It has been suggested that a value of $8.0 \mu \mathrm{g}$ per activity unit is representative of many heparin preparations (Jaques et al., 1990). It is clear that for a drug as widely used as heparin, it is of crucial importance to ensure that measurements of biological activity are properly standardized. It is for this reason that the biological activity of every commercial heparin preparation is determined by comparison with the International Standard heparin preparation, of which the activity is defined as 1370 Units/ampoule (Barrowcliffe, 1989). Unlike other drugs in other systems, the activity for a given commercial preparation relative to the International Standard heparin preparation changes depending on the method of comparison in the different blood coagulation tests used. Hence, the values will not necessarily be the same for any two identical preparations compared using different procedures. 


\section{Monitoring heparin treatments}

A heparin treatment must result in blood heparin levels that are sufficient to control thrombosis and yet avoid undue risk of bleeding. However, the antithrombotic effect and the risk of bleeding varies, not only with the dose, but also with the individual patient response. This variation in response to heparin calls for individualization of the heparin dose regimen and a careful clinical monitoring by laboratory tests (Abildgaard, 1989). The activated partial thromboplastin time (APTT) is the most widely used clinical laboratory test for heparin monitoring. In this test, the clotting time of a plasma sample is measured after the addition of an initiator of the blood clotting process. In the presence of this initiator, coagulation is greatly accelerated and anticoagulant activity can then be measured by the delay of appearance of a clot, with clotting times between 1 and $2 \mathrm{~min}$. Unfortunately, the reagents and methodology used to perform APTT assays vary widely between different laboratories. For normal plasma, a typical value of the APTT is of the order of 30 to $40 \mathrm{~s}$. Usually a therapeutic range of $1.5-2.5$ times the mean value of the normal reference range is recommended. By translating this APTT range to therapeutic heparin concentration ranges, this has been defined as 0.2-0.7 Units/ml (Van Putten et al., 1982) or more narrowly as $0.3-0.5 \mathrm{Units} / \mathrm{ml}$ (Hirsch, 1986). It must be noted however, that the clotting time determined by an APTT assay is not only dependent on the amount of active heparin, but also on the concentration of AtIII and other coagulation factors.

A more specific test for heparin is a chromogenic substrate test. These tests are specific peptide substrate tests, which are available for thrombin as well as for factor Xa. The principle of these tests is the determination of residual enzyme activity after the addition of a fixed quantity of thrombin or factor $\mathrm{Xa}$ to the heparinized plasma sample and a defined incubation time. During this incubation time some of the thrombin or factor $\mathrm{Xa}$ will be inactivated by AtIII, depending on the amount of active heparin available. Residual thrombin or factor $\mathrm{Xa}$ is determined either by the addition of peptide substrate to the mixture or by subsampling a sample from the reaction mixture into the substrate solution. All the commercially available substrates use p-nitroanaline as a chromophore, which is released as a result of the reaction with thrombin or factor $\mathrm{Xa}$. This release is measured by monitoring the change in optical density at $405 \mathrm{~nm}$. If the test plasma is the only source of antithrombin, the result is influenced by the AtIII concentration, which can be different from patient to patient, and so a combined heparin/ antithrombin activity is measured (Teien \& Lie, 1975; Bärtl et al., 1980). If AtIII is added as a reagent to the test sample, variations in the plasma AtIII concentration of the test sample will have minimal influence on the result (Teien \& Lie, 1977), which in this case is only dependent on the concentration of active heparin in the plasma.

In clinical practice no consensus of opinion exists concerning which is the most useful test to monitor a heparin treatment. It is still unclear whether the concentration of active heparin or the total "clotting status" (as determined by a clotting assay, and dependent on several other parameters) is the most important parameter (Holm et al., 1987). Patients with low plasma AtIII concentration may still have (too) low anticoagulant activity, even if the active heparin concentration is well within what is usually considered as the "therapeutic range". On the other hand, if only the clotting time of patients with low AtIII concentration is measured, a defined therapeutic range may lead to excessive heparin dosage and an undue risk of bleeding. Moreover, it is found that the plasma AtIII concentration regularly decreases during heparin treatment (Holm et al., 1985).

Another important aspect to this discussion is the observation of several research groups that when injected in vivo heparin generates (extra) anticoagulant activity. When heparin was injected and subsequently separated from the blood, the separated heparin had higher anticoagulant activity per $\mathrm{mg}$ than the same heparin before injection (Levy \& Jacques, 1978, Björnsson \& Wolfram, 1982, Klein et al.,1982). This observation indicates important differences between the measurement of absolute concentrations of heparin and the "amount of heparin present" as determined by an in vitro coagulation test based on a calibration curve. Hence Jaques et al., (1990) claim that it is essential to have a method to determine the total absolute amount of heparin in plasma. 
An ISFET based heparin sensor using the ionstep measuring method

An aspect of current heparin assays is that they each use additional reagents and specific equipment and can only be performed in the laboratory by skilled personnel. A simple sensor system, that can measure directly in a drop of whole blood, might therefore be a useful device for heparin monitoring. The application of such a sensor requires only very small samples (in the order of microlitres). If necessary, blood cells and platelets could be directly excluded by using a filter membrane. Because no sample pretreatment is necessary and no additional reagents are used, a simple heparin sensor system might be used at the "bedside" and therefore save time and money. It is interesting to note in this context that approximately 500 million doses of heparin are used worldwide each year (Linhardt, 1991), which means that at least some tens of millions of tests are performed ( $\$ 5-\$ 10$ per test).

In a previous paper we introduced an ISFETbased heparin sensor system using the ion-step measuring method (Van Kerkhof et al., 1993). The principle of this measuring method was to detect a change in charge density in a porous membrane which was mounted on top of an ISFET (Schasfoort et al., 1990). For the specific binding of heparin in the membrane, protamine was used as an affinity ligand. Protamine sulphate is used clinically to neutralize heparin and to counteract its anticoagulant effects. Protamine is a very basic (alkaline) protein with a molecular weight of about 4000 , typically containing 20 arginines out of 30 amino acids. It has been found that the interaction of protamine and heparin consists of a 1:1 pairing of anionic heparin sites with cationic protamine sites (Cundall et al., 1982). By immobilizing protamine as an affinity ligand in this membrane interactions between protamine and heparin can be detected because these interactions induce a change in the charge density in the membrane. Because protamine binds all heparin molecules, independent of their anticoagulant activity, the sensor system measures total concentrations of heparin.

To determine the charge density in the membrane, the ISFET with the membrane is placed in a wall-jet cell of a flow-system and an equilibrium is established in $10 \mathrm{mM} \mathrm{KCl}$. Subsequently, the solution which is in contact with the ISFET is suddenly replaced (within $100 \mathrm{~ms}$ ) by a solution with a $\mathrm{KCl}$ concentration of 50 or $100 \mathrm{mM}$ with the same $\mathrm{pH}$. As a response to this so-called "ion-step" (at constant $\mathrm{pH}$ ) the ISFET amplifier shows a transient output voltage which eventually returns to its original value. The amplitude of this voltage peak is a measure of the charge density and thus the amount of bound molecules in the membrane.

The ISFET itself also shows a response to an ion-step at constant $\mathrm{pH}$ and this response sometimes interferes with the membrane response. Therefore, such ISFET responses were investigated in more detail and the mechanism was elucidated (Van Kerkhof et al., 1994a). As a consequence of the results of this investigation, the possibility of direct modification of the ISFET surface was investigated with a view to obtaining a surface with high affinity for heparin. The first successful result was obtained from a treatment with an amino functionalized silane coupling agent. This results in a coverage with a covalently bound, positively charged layer of polysiloxanes to which heparin binds through electrostatic interaction (Van Kerkhof et al., 1994b). However, a better result is achieved by direct immobilization of protamine at the ISFET surface, as described in this paper. The immobilized protamine acts as an affinity ligand for heparin and the interaction can be detected by the ion-step response of the ISFET.

To the knowledge of the authors, only one other research group has reported the development of a heparin concentration sensor (Ma et al., 1992, Yang et al., 1993, Ma et al., 1993). This sensor was based on an ion-selective electrode with a polymer membrane, in which a quaternary ammonium salt was immobilized that showed a significant potentiometric response to heparin. The exact mechanism responsible for this response is still unclear. The concentration range which could be determined in blood plasma was reported as 1.0 to $9.8 \mathrm{Units} / \mathrm{ml}$. The sensor can therefore be used in surgical procedures where doses of 2 to 8 Units $/ \mathrm{ml}$ are commonly used. However, in treatment of post-operative thrombosis and embolism, the therapeutic concentration range is 0.2 to $0.7 \mathrm{Units} / \mathrm{ml}$ which means that this sensor can not be used as an alternative for the current assay methods. Therefore, the development of a heparin sensor that also covers this therapeutic range is still necessary. 


\section{MATERIALS AND METHODS}

\section{Reagents}

Protamine sulphate (grade $\mathrm{X}$, from salmon) was purchased from Sigma Chemical Co. and heparin from Organon Teknika (Thromboliquine ${ }^{\circledR}, 5 \mathrm{ml}$ ampoules containing 25000 Units). For some of the experiments, the heparin used was made in the pharmacy of the "Medisch Spectrum Twente (MST)" hospital (sodium salt, $5 \mathrm{ml}$ ampoules, 5000 Units/ml); this heparin will be referred to as "MST heparin". Because the heparin concentration of both heparin preparations are defined in Units $/ \mathrm{ml}$, the heparin concentrations in this paper are also given in Units $/ \mathrm{ml}$, although the response of the sensor is related to absolute concentrations. Phosphate buffer was a mixture of $\mathrm{Na}_{2} \mathrm{HPO}_{4}$ and $\mathrm{NaH}_{2} \mathrm{PO}_{4}$ (both products of Merck) in the appropriate ratio. HEPES buffer was a product of Aldrich Chemical Co. and phosphate buffered saline (PBS) tablets were purchased from Sigma Chemical Co. Normal plasma samples (platelet free, from a pool of a large group of healthy donors) were obtained from the laboratory of the MST hospital, along with the plasma samples of different heparinized patients of which the APTT was determined using a kaolin/cephalin APTT assay (Diagnostica Stago).

\section{Measurement set-up}

The measurement set-up consisted of a flowsystem in which the flow was controlled by an effective pressure $(0.1$ bar) of nitrogen in the two vessels containing the solutions. The resulting flow rate was $1.3 \mathrm{ml} / \mathrm{min}$. The ISFET was mounted in a wall-jet cell, in which the liquid flow was perpendicular to the ISFET surface. A cross-section of the wall-jet cell is shown in Fig. 1. The electrolyte at the surface of the ISFET could be replaced within about $0 \cdot 1 \mathrm{~s}$ (ion-step). A saturated calomel electrode was used to define the potential of the solution. In all experiments described in this paper, $10 \mathrm{mM}$ and $100 \mathrm{mM} \mathrm{KCl}$ solutions were used as the "ion-step" solutions. The solutions were buffered with $0.2 \mathrm{mM} \mathrm{HEPES}$ or $0.1 \mathrm{mM}$ phosphate buffer. The responses of the ISFET, connected to a source drain follower, were recorded with a Nicolet 310 digital oscilloscope, which can store recorded curves on a floppy disk. The output potential of the source-

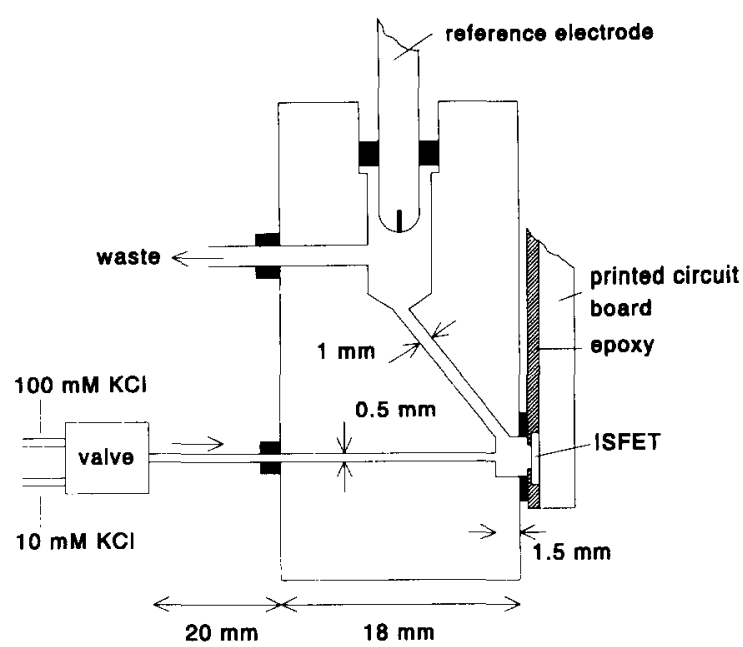

Fig. 1. Cross-section of the wall-jet cell of the flow through system.

drain follower in the case of the equilibrium situation in $10 \mathrm{mM} \mathrm{KCl}$ was defined as $0 \mathrm{mV}$. For representation purposes, the curves were filtered with a software low-pass filter using a cut-off frequency of $40 \mathrm{~Hz}$.

\section{Measurement devices}

ISFETs with a $\mathrm{Ta}_{2} \mathrm{O}_{5}$ gate insulator were fabricated in the MESA cleanroom laboratory following the usual ISFET processing steps. The ISFETs showed a $\mathrm{pH}$ response of $-59 \mathrm{mV} / \mathrm{pH}$. The protamine was immobilized by physical adsorption. The ISFETs were immersed in a solution of $10 \mathrm{mg} / \mathrm{ml}$ protamine sulphate in PBS for at least $24 \mathrm{~h}$, rinsed in $4 \mathrm{M} \mathrm{NaCl}$ and subsequently stored in a PBS solution.

\section{Measurement protocol}

Before each incubation period in a heparin containing sample, the ISFETs with the immobilized protamine were characterized by their response to an ion-step of 10 to $100 \mathrm{mM} \mathrm{KCl}$. Each time the ion-step response had to be determined, 3 to 5 responses were successively recorded without removing the ISFET, and the amplitude of the ion-step response was defined as the mean value of the different responses.

For the determination of heparin concentrations in PBS solutions, small $15 \mathrm{ml}$ vessels were used, in which the ISFET was placed for two min, during which time the solution was not stirred. After incubation, the ISFET was rinsed 
with PBS and mounted in the wall-jet cell of the measurement set-up. The ion-step response was recorded and the change in the amplitude, with respect to the response before incubation, was determined. The ISFETs with the protamine layer could be regenerated after each measurement in a heparin solution, by rinsing for about $1 \mathrm{~min}$ in a $4 \mathrm{M} \mathrm{NaCl}$ solution, followed by an equilibration period in a PBS solution (varying from 5 min to $1 \mathrm{~h}$ ). When recording a calibration curve, which gives the change of the ion-step response as a function of the heparin concentration, only one ISFET was used, which was regenerated after each measurement.

For the determination of heparin concentrations in normal plasma, a test-tube containing $2 \mathrm{ml}$ of normal plasma was used, to which small amounts of a 100 Units $/ \mathrm{ml}$ heparin solution (in $0.9 \% \mathrm{NaCl}$ ) were added to obtain the different concentrations. The ISFET was incubated in the plasma for 2 min (without stirring, with a view to future applications where only a drop of blood is needed), rinsed in PBS and subsequently the ion-step response was determined. The heparin concentration of the plasma samples of the different heparinized patients was also determined by placing the ISFET in a test-tube containing 0.5 or $1.0 \mathrm{ml}$ of the plasma sample. After $2 \mathrm{~min}$ the ISFET was rinsed with PBS and the change in the amplitude of the ion-step response, with respect to the response before incubation, was determined. Then $5 \mu \mathrm{l}$ of a $50 \mathrm{Units} / \mathrm{ml}$ or 100 Units/ml heparin solution was added to yield an increase in the heparin concentration of 0.5 Units $/ \mathrm{ml}$. After regeneration in a $4 \mathrm{M} \mathrm{NaCl}$ solution, the ISFET was used to measure this increased heparin concentration, following the same procedure.

\section{RESULTS}

Figure 2 shows some typical ion-step responses to 10 to $100 \mathrm{mM} \mathrm{KCl}$ at $\mathrm{pH} 7 \cdot 0$. Curve 1 represents a typical ion-step response of a "bare" $\mathrm{Ta}_{2} \mathrm{O}_{5}$-ISFET without a protamine layer. Curve 2 represents a typical ion-step response of an ISFET with an adsorbed layer of protamine at the gate oxide surface and curve 3 is a typical ion-step response of an ISFET with a protamine layer after 2 min of incubation in a PBS solution containing 0.9 Units $/ \mathrm{ml}$ heparin. The change in the amplitude of the ion-step response after

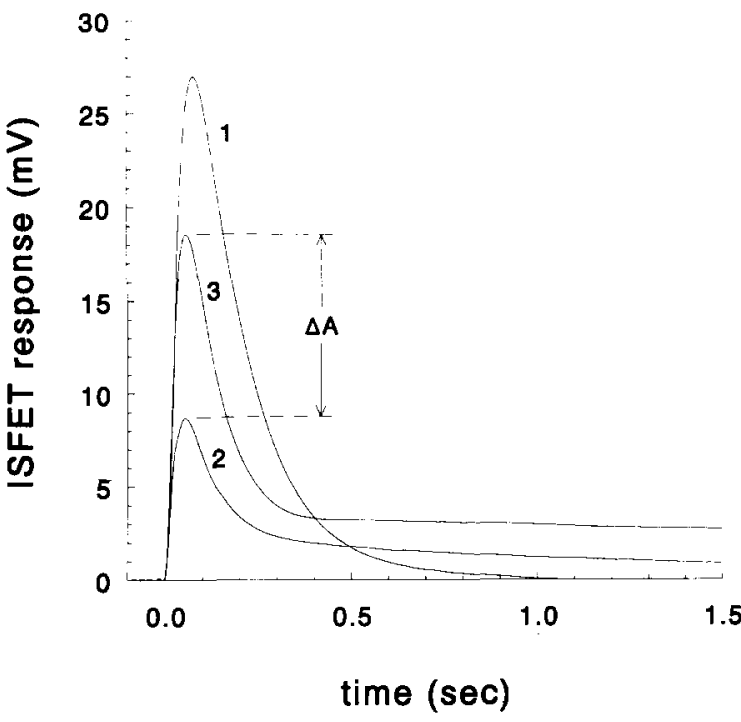

Fig. 2. Typical responses to an ion-step of 10 to $100 \mathrm{mM} \mathrm{KCl}$ at $\mathrm{pH} \mathrm{7.0}$. Curve 1 represents a bare ISFET, curve 2 an ISFET with a protamine layer and curve 3 an ISFET with a protamine layer after incubation in PBS containing 0.9 Units $/ \mathrm{ml}$ heparin. $\triangle A$ is the change in amplitude after incubation in a heparin solution.

incubation (curve 3) with respect to the response before incubation (curve 2) is referred to as $\Delta \mathrm{A}$. The $\mathrm{KCl}$ solutions used for recording curve 1 were buffered with $0.2 \mathrm{mM}$ HEPES, whereas the solutions used for recording curve 2 and 3 , were buffered with $0 \cdot 1 \mathrm{mM}$ phosphate buffer. It appeared however, that using $0.2 \mathrm{mM}$ HEPES instead of $0.1 \mathrm{mM}$ phosphate buffer (or vice versa) did not significantly change the response. Because the $\mathrm{pH}$ of the 10 and $100 \mathrm{mM} \mathrm{KCl}$ solutions were not exactly the same, curve 2 and 3 do not return to $0 \mathrm{mV}$ for $\mathrm{t} \rightarrow \infty$. ISFETs with an adsorbed protamine layer could be stored in PBS for at least two weeks without any change in the ion-step response at $\mathrm{pH} 7$.

In Fig. 3, $\Delta \mathrm{A}$ is plotted as a function of the heparin concentration in a PBS solution. The $\mathrm{KCl}$ solutions were buffered with $0.1 \mathrm{mM}$ phosphate buffer at $\mathrm{pH} 7.0$ and the ISFET was incubated for 2 mins in the heparin solution. In this case Thromboliquine ${ }^{\circledR}$ heparin was used. The difference between the amplitudes of the 3 to 5 different responses recorded for each measurement was about $0.4 \mathrm{mV}$ (mean value $\pm 0.2 \mathrm{mV}$ ). Because the parameter $\Delta \mathrm{A}$, which is shown in Fig. 3 , is the difference between the mean value $( \pm 0.2 \mathrm{mV})$ of the response before incubation and the mean value $( \pm 0.2 \mathrm{mV})$ of the response 


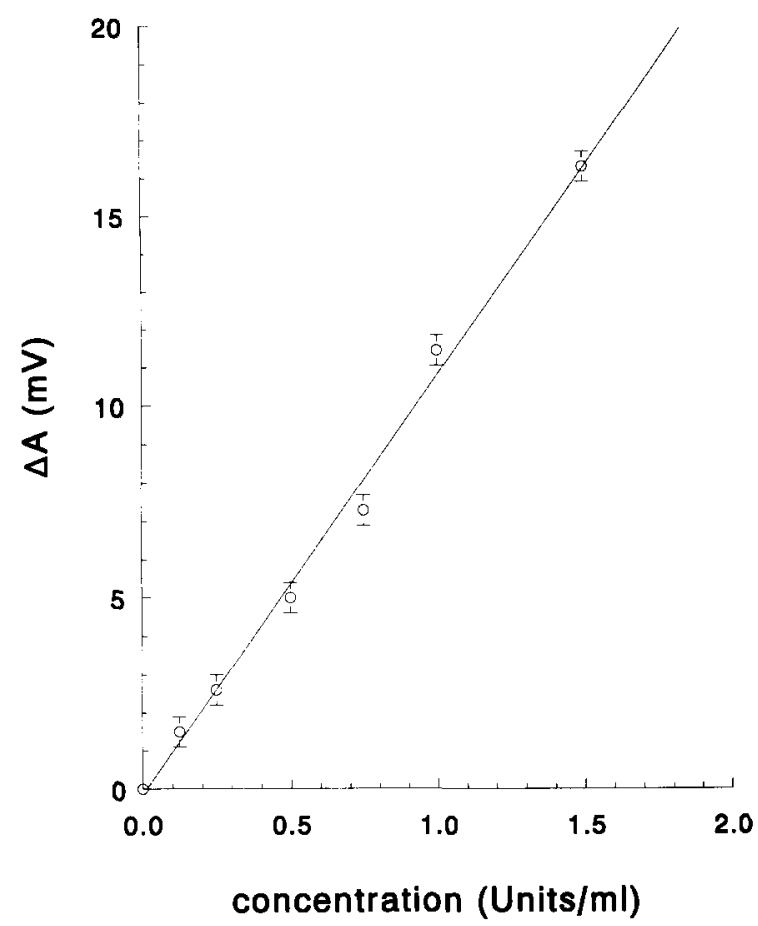

Fig. 3. The change in amplitude of the ion-step response $\triangle A$ after 2 minutes incubation in a PBS solution as a function of the heparin concentration (ISFET A).

after incubation, the accuracy of this parameter is $\pm 0.4 \mathrm{mV}$, as indicated by the error bars. The ISFET was regenerated after each measurement by rinsing with $4 \mathrm{M} \mathrm{NaCl}$ and used again for the following measurement.

The relation between the change in amplitude, $\Delta \mathrm{A}(\mathrm{mv})$, of the ion-step response and the heparin concentration in PBS, $\mathrm{c}_{\mathrm{b}}$ (Units $\left./ \mathrm{ml}\right)$, can be described by a linear curve fit of the data points as shown in Fig. 3. The linear equation is given in Table 1 (ISFET code A), together with the linear equations of the curve fit of three other ISFETs with a protamine layer, which were used to determine $\Delta \mathrm{A}$ as a function of the heparin concentration in PBS.

Figure 4 shows $\Delta \mathrm{A}(10$ to $100 \mathrm{mM} \mathrm{KCl}$, $0.1 \mathrm{mM}$ phosphate buffer, $\mathrm{pH} 7 \cdot 0$ ) as a function of the heparin (Thromboliquine ${ }^{\circledR}$ ) concentration in normal plasma, in which the ISFET was incubated for $2 \mathrm{~min}$. The same ISFET (code A) and the same solutions were used as for the measurement of the heparin concentration in PBS. The error bars indicate the accuracy of $\Delta \mathrm{A}$ as explained in the legend to Fig. 3. The relation between the change in amplitude, $\Delta \mathrm{A}(\mathrm{mV})$, and the heparin concentration in plasma, $c_{p}$ (Units/ $\mathrm{ml}$ ), can again be described by a linear curve fit of the data points, starting from 0.25 Units $/ \mathrm{ml}$, and the linear equation is given in Table 1 (code A). Note the difference in offset and slope in comparison with the curve fit of the measurements in PBS. The ISFET with code B was also used to determine heparin concentrations in normal plasma and the linear equation of the curve fit of the results is given in Table 1. For this ISFET, also note the difference in offset and slope in comparison with the curve fit of the measurements in PBS.

Figure 5 shows $\Delta \mathrm{A}$ as a function of the heparin concentration in normal plasma, determined with another ISFET (code D). In this case the ionstep responses were recorded at $\mathrm{pH} 7.4$ and the $\mathrm{KCl}$ solutions were buffered with $0.2 \mathrm{mM}$ HEPES. MST heparin was used. The linear curve fit is again given in Table 1 . This curve was used as a calibration curve for the measurement of the heparin concentration in plasma samples of several patients who where heparinized with MST heparin. In Table 2 the heparin concentrations of these samples are presented as determined with the ion-step measuring method using Fig. 5 as a calibration curve. After each measurement of the heparin concentration of a specific sample, $0.5 \mathrm{Units} / \mathrm{ml}$ heparin were added to the sample and the concentration was measured again. These values are also given in Table 2 . Thirteen different samples were tested, and in nine of the samples the increase in the heparin concentration of 0.5 Units $/ \mathrm{ml}$ was correctly measured within the range $0.5 \pm 0.07$ Units $/ \mathrm{ml}$. In the other 4 samples the measured increase was less than the added 0.5 Units/ml.

The ISFET used for these experiments (code D) was regenerated more than fifty times. The amplitude of the calibration response, which was recorded after each regeneration procedure, only changed by about $2 \mathrm{mV}$.

Table 2 also shows the values for the APTT of the different plasma samples. Figure 6 shows the relation between the APTT and the measured heparin concentrations for the nine samples in which the sensor correctly measured the increase of 0.5 Units $/ \mathrm{ml}$ as well as for the other 4 samples. It can be seen that no clear relationship exists between the APTT value and the measured heparin concentration. 
TABLE 1 Linear curve fits of heparin concentration measurements of different ISFETs. The ion-step responses of ISFET D were recorded at $\mathrm{pH} 7.4$ and MST heparin was used. The other ISFETs were measured at $\mathrm{pH} 7.0$ and Thromboliquine was used as heparin. $\Delta \mathrm{A}$ is the change in amplitude after incubation $(\mathrm{mV}), \mathrm{c}_{\mathrm{b}}$ is the heparin concentration in PBS and $c_{p}$ the heparin concentration in normal plasma (both in units $/ \mathrm{ml}$ ).

$\begin{array}{lcc}\begin{array}{l}\text { ISFET } \\ \text { code }\end{array} & \begin{array}{c}\text { Linear curve fit of heparin } \\ \text { concentration measurement } \\ \text { in PBS }\end{array} & \begin{array}{c}\text { Linear curve fit of heparin } \\ \text { concentration measurement } \\ \text { in normal plasma }\end{array}\end{array}$

\begin{tabular}{llc}
\hline A & $\Delta \mathrm{A}=-0 \cdot 15+11 \cdot 0 \mathrm{c}_{\mathrm{b}}$ (fig. 2) & $\Delta \mathrm{A}=2 \cdot 53+6 \cdot 10 \mathrm{c}_{\mathrm{p}}$ (Fig. 3) \\
$\mathrm{B}$ & $\Delta \mathrm{A}=-0 \cdot 30+10 \cdot 6 \mathrm{c}_{\mathrm{b}}$ & $\Delta \mathrm{A}=2 \cdot 38+4 \cdot 51 \mathrm{c}_{\mathrm{p}}$ \\
$\mathrm{C}$ & $\Delta \mathrm{A}=-0 \cdot 25+11 \cdot 6 \mathrm{c}_{\mathrm{b}}$ & - \\
$\mathrm{D}$ & $\Delta \mathrm{A}=-0 \cdot 01+11 \cdot 3 \mathrm{c}_{\mathrm{b}}$ & $\Delta \mathrm{A}=3 \cdot 17+4 \cdot 86 \mathrm{c}_{\mathrm{p}}$ (Fig. 4) \\
\hline
\end{tabular}

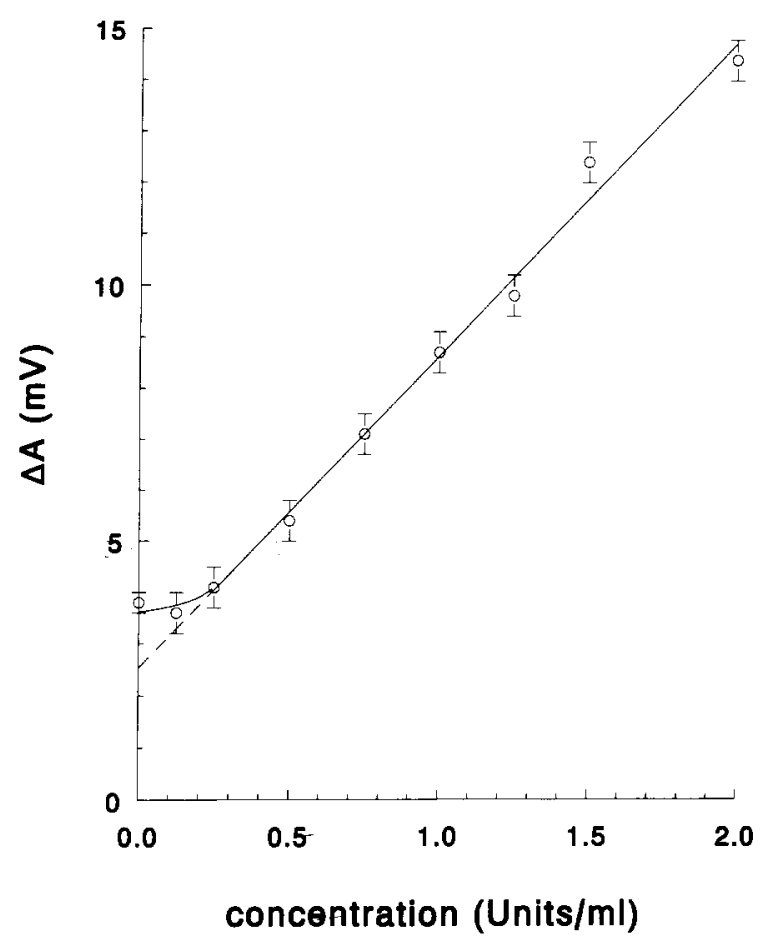

Fig. 4. The change in amplitude of the ion-step response $\Delta A$ after 2 minutes incubation in normal plasma as a function of the heparin concentration (ISFET A).

\section{DISCUSSION AND CONCLUSIONS}

The mechanism behind the ion-step response of a "bare" ISFET as shown in Fig. 2, curve 1, has already been described (Van Kerkhof, 1994a) but will be briefly reviewed.

The interface between the ISFET and the solution is described by the acid-base equilibria at the amphoteric surface of the $\mathrm{Ta}_{2} \mathrm{O}_{5}$-gate

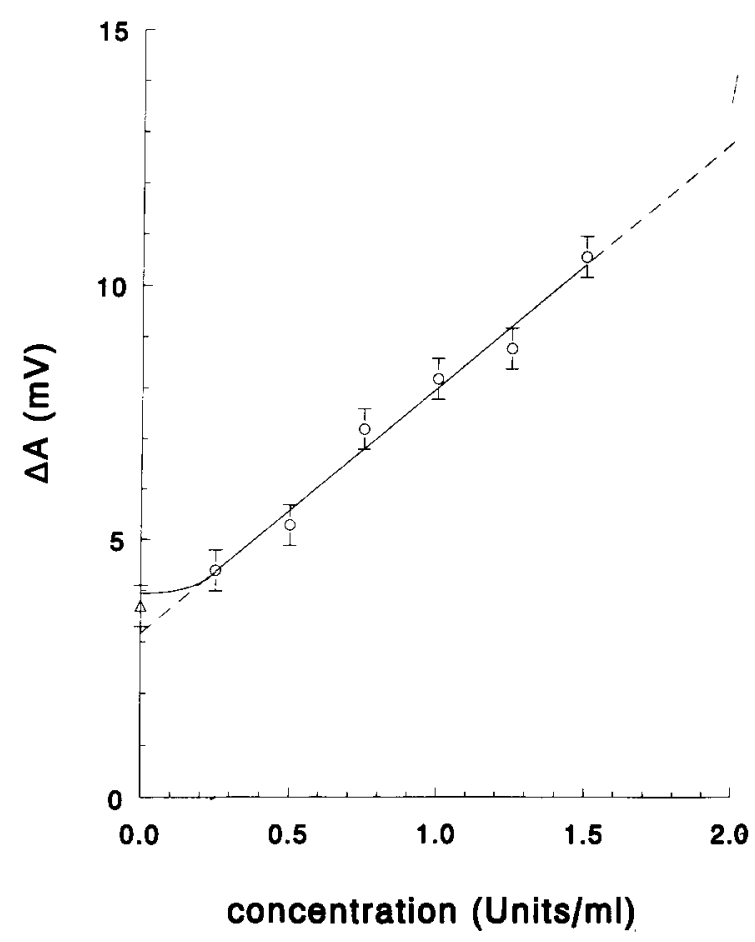

Fig. 5. The change in amplitude of the ion-step response $\Delta A$ after 2 minutes incubation in normal plasma as a function of the heparin concentration (ISFET D). Calibration curve for the experiments shown in Table 2.

oxide. The surface $\mathrm{OH}$ groups can act as proton donors as well as proton acceptors, yielding a charge density, $\sigma$ (in $\mathrm{C} / \mathrm{cm}^{2}$ ), at the surface.

$$
\sigma=\mathrm{q}\left(\left[\mathrm{Ta}-\mathrm{OH}_{2}^{+}\right]-\left[\mathrm{Ta}-\mathrm{O}^{-}\right]\right)
$$

The $\mathrm{pH}$ where $\sigma=0$ is called the point of zero charge, $\mathrm{pH}_{\mathrm{pzc}}$, which for $\mathrm{Ta}_{2} \mathrm{O}_{5}$ lies around $\mathrm{pH}$ 
TABLE 2 Results of measurements in plasma samples of heparinized patients.

\begin{tabular}{ll}
$\begin{array}{l}\text { Measured } \\
\text { heparin }\end{array}$ & $\begin{array}{l}\text { Measured change } \\
\text { (in Units } / \mathrm{ml} \text { ) }\end{array}$ \\
$\begin{array}{l}\text { concentration } \\
\text { (Units } / \mathrm{ml} \text { ) }\end{array}$ & $\begin{array}{c}\text { after addition of } \\
0.5 \mathrm{Units} / \mathrm{ml}\end{array}$ \\
\hline
\end{tabular}

\begin{tabular}{lrr}
\hline 1.18 & 0.47 & 141 \\
1.22 & 0.51 & 86 \\
1.00 & 0.45 & 144 \\
0.85 & 0.49 & 156 \\
1.30 & 0.47 & 73 \\
1.00 & 0.47 & 92 \\
1.02 & 0.43 & 55 \\
0.79 & 0.54 & 93 \\
0.97 & 0.43 & 87 \\
1.06 & 0.35 & 134 \\
1.06 & 0.37 & 67 \\
0.75 & 0.23 & 72 \\
0.95 & 0.27 & 119 \\
\hline
\end{tabular}

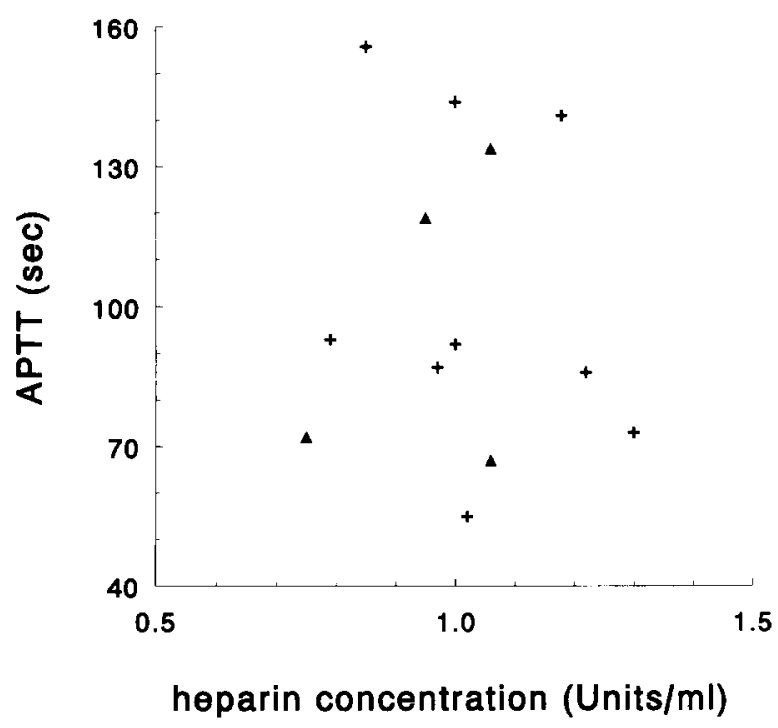

Fig. 6. The APTT as function of the measured heparin concentration for the different plasma samples of heparinized patients. The crosses (+) represent the samples in which the addition of 0.5 Units $/ \mathrm{ml}$ was correctly measured, and the triangles $(\mathbf{\Delta})$ represent the other samples.

3 ; this means that at $\mathrm{pH} 7$ the $\mathrm{Ta}_{2} \mathrm{O}_{5}$ surface is negatively charged. The relation between the surface charge, $\sigma$, and the surface potential, $\Psi$, is given by:

$$
\sigma=\Psi \mathrm{C}_{\mathrm{dl}}
$$

where $\mathrm{C}_{\mathrm{dl}}$ is the double layer capacitance (in $\mathrm{F}$ / $\mathrm{cm}^{2}$ ).

The ISFET measures the surface potential, $\Psi$, with respect to the bulk potential, which is defined by the reference electrode. This potential $\Psi$ follows from the Boltzmann equation:

$$
\left[\mathrm{H}_{\mathrm{s}}^{+}\right]=\left[\mathrm{H}_{\mathrm{b}}^{+}\right] \exp \left(\frac{-\mathrm{q} \Psi}{\mathrm{kT}}\right)
$$

where $\left[\mathrm{H}_{\mathrm{s}}^{+}\right]$is the proton concentration at the ISFET surface and $\left[\mathrm{H}_{b}^{+}\right]$the proton concentration in bulk solution. The combination of the large number of $\mathrm{OH}$ surface sites (about $10^{15} / \mathrm{cm}^{2}$ ), the chemical equilibria of the acid-base reactions and the relative low value of the double layer capacitance (maximum $20 \mu \mathrm{F} / \mathrm{cm} 2$ ), makes the $\mathrm{Ta}_{2} \mathrm{O}_{5}$ oxide surface act as a very good buffer for $\mathrm{H}_{\mathrm{s}}^{+}$, which results in an almost constant $\mathrm{pH}$ at the surface $\left(\mathrm{pH}_{\mathrm{s}}\right)$. Consequently, the surface potential, $\Psi$, changes with $-59 \mathrm{mV} / \mathrm{pH}_{\mathrm{b}}$ (van Hal et al., 1994).

Equations 1 to 3 describe the statical behaviour of the ISFET in thermodynamical equilibrium. However, the transient response to an ion-step cannot directly be described by these equations. To elucidate the mechanism behind these responses, a dynamical simulation model was developed based on the Nernst-Planck and Poisson equations, which are solved by a finite difference procedure. The chemical equilibria at the ISFET surface are incorporated in this model of the ISFET, together with a stagnant layer in which ion-transport is caused only by diffusion and migration. With this model it is possible to understand the dynamic mechanism and verify the experimental results (Van Kerkhof et al., 1994a).

After an ion-step, $\mathrm{C}_{\mathrm{dl}}$ increases very fast due to a sudden increase in the diffuse capacitance, which results in a decrease in the absolute value of the surface potential $\Psi$, according to eqn. (2). According to the Boltzmann equation (3), the $\mathrm{H}_{\mathrm{s}}^{+}$concentration will tend to decrease because of the decreased $\Psi$. However, the oxide surface acts as a very good buffer for $\mathrm{H}_{\mathrm{s}}^{+}$and will keep the $\mathrm{H}_{\mathrm{s}}^{+}$concentration constant by dissociating $\mathrm{Ta}-\mathrm{OH}$ groups. This will change $\sigma$ until a new equilibrium is reached, when $\sigma / \mathrm{C}_{\mathrm{dl}}(=\Psi)$ has the same value as before the ion-step. The time constant of adapting $\sigma$ by dissociating $\mathrm{Ta}-\mathrm{OH}$ groups is determined by the diffusion of the $\mathrm{H}^{+}$ ions and the buffer capacity of the electrolyte. A thick stagnant layer and/or a low buffer 
capacity will delay the establishment of a new equilibrium. This was indeed observed in experiments that were in agreement with the simulation results.

If the double layer capacitance changes, as a result of an ion-step of 10 to $100 \mathrm{mM} \mathrm{KCl}$, from $\mathrm{C}_{\mathrm{d} 11}$ to $\mathrm{C}_{\mathrm{d} 12}$, the theoretical maximum change in $\Psi$ directly after the ion-step (assuming that $\sigma$ is not changing yet) is:

$$
\begin{aligned}
\Delta \Psi_{\max } & =\Psi_{2}-\Psi_{1}=\frac{\sigma}{\mathrm{C}_{\mathrm{d} 12}}-\frac{\sigma}{\mathrm{C}_{\mathrm{d} 11}} \\
& =\psi_{1}\left(\frac{\mathrm{C}_{\mathrm{d} 11}}{\mathrm{C}_{\mathrm{d} 12}}-1\right)
\end{aligned}
$$

The double layer capacitance can be calculated using the Gouy-Chapman-Stern model and depends on the electrolyte concentration and on the potential $\Psi$ across the double layer. Figure 7 show the values of $C_{d 11}$ (in $10 \mathrm{mM}$ ) and $C_{d 12}$ (in $100 \mathrm{mM}$ ), as well as the ratio $\mathrm{C}_{\mathrm{dl1}} / \mathrm{C}_{\mathrm{dl} 2}$, as a function of the potential $\Psi$, calculated according to the Gouy-Chapman-Stern model; a constant Stern capacity of $20 \mu \mathrm{F} / \mathrm{cm}^{2}$ is assumed (Bard \& Faulkner, 1980).

At $\mathrm{pH} 7$, the surface potential, $\Psi$, of a $\mathrm{Ta}_{2} \mathrm{O}_{5}$ ISFET $\left(\mathrm{pH}_{\mathrm{pzc}}=3\right)$ is $(7-3) \times-59=-236 \mathrm{mV}$. The double layer capacitance at $10 \mathrm{mM} \mathrm{KCl}$ is $12.7 \mu \mathrm{F} / \mathrm{cm}^{2}$, which results, according to eqn. 2 in a surface charge, $\sigma$, of $-3.00 \times 10^{-6} \mathrm{C} /$ $\mathrm{cm}^{2}$, corresponding with $1.87 \times 10^{13}$ negatively charged groups per $\mathrm{cm}^{2}$. The value of $C_{d l}$ at

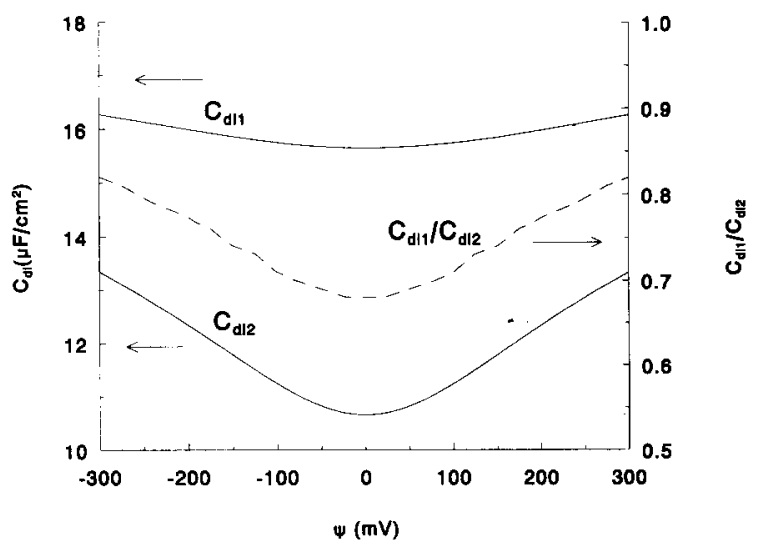

Fig. 7. The double layer capacitance, $C_{\mathrm{d} b}$, as a function of the potential $\Psi$ across the double layer. $C_{\mathrm{d} 11}$ is the capacitance in $10 \mathrm{mM} \mathrm{KCl}, C_{\mathrm{d} 12}$ the capacitance in $100 \mathrm{mM} \mathrm{KCl}$ (left $Y$-axis) and $C_{\mathrm{d} 11} / C_{\mathrm{dl} 2}$ represents the ratio of the two capacitances as used in equation 4 (right $Y$-axis).
$100 \mathrm{mM} \mathrm{KCl}$ is $16 \cdot 1 \mu \mathrm{F} / \mathrm{cm}^{2}$, which gives a value of $50 \mathrm{mV}$ for $\Delta \Psi_{\max }$ as defined by eqn. 4 .

Because of an insufficient time separation between the changes in $\mathrm{C}_{\mathrm{dl}}$ and $\sigma$, which is caused by the stagnant layer related to the buffer capacity of the solution, the real value of the amplitude of the ion-step response is smaller than the theoretical maximum. This effect was clearly observed in the simulation results (Van Kerkhof et al., 1994a). The experimental result of $27 \mathrm{mV}$ (Fig. 2) implies that the response reaches $54 \%$ of its maximum value due to the insufficient time separation between the changes in $\mathrm{C}_{\mathrm{d} l}$ and $\sigma$.

Immobilization of protamine by physical adsorption will result in a coverage with a maximum thickness of a few monolayers, but since the ISFETs are rinsed in $4 \mathrm{M} \mathrm{NaCl}$, a monolayer coverage seems most plausible. Due to the small size of the protamine molecules (MW 4000), the protamine layer will be very thin; this makes it acceptable to assume that the immobilization of a protamine layer can be described as a modification of the surface charge of the ISFET. The ion-step response can therefore be described in the same way as the ion-step response of a bare ISFET, but with a different surface charge density, $\sigma$, and point of zero charge, $\mathrm{pH}_{\mathrm{pzc}}$. Therefore, the contribution of socalled membrane effects, which were described in a previous description of the heparin sensor (Van Kerkhof et al., 1993), can be neglected. These effects include a changing Donnan potential at the protamine-solution interface, and a temporary increase in the $\mathrm{pH}$ inside the protamine layer as a result of an uptake of protons by the protamine molecules induced by the stepwise increase in the electrolyte concentration.

The measured ion-step response of an ISFET with a protamine layer, as shown in Fig. 2, curve 2 , supports the assumption to describe the effect of a protamine layer coverage as a surface modification. The ion-step has a smaller amplitude due to the positive charge of the protamine, compensating a part of the negative charge of the $\mathrm{Ta}_{2} \mathrm{O}_{5}$, but the time constants of the ionstep responses shown in Fig. 2 are about the same. This is shown in Fig. 8, where the responses of Fig. 2 are shown again but in this case are normalized to an amplitude of 1 . It can be seen that the time constants are all comparable, including those of curve 3 , which represents the response after incubation in heparin. Apparently, 


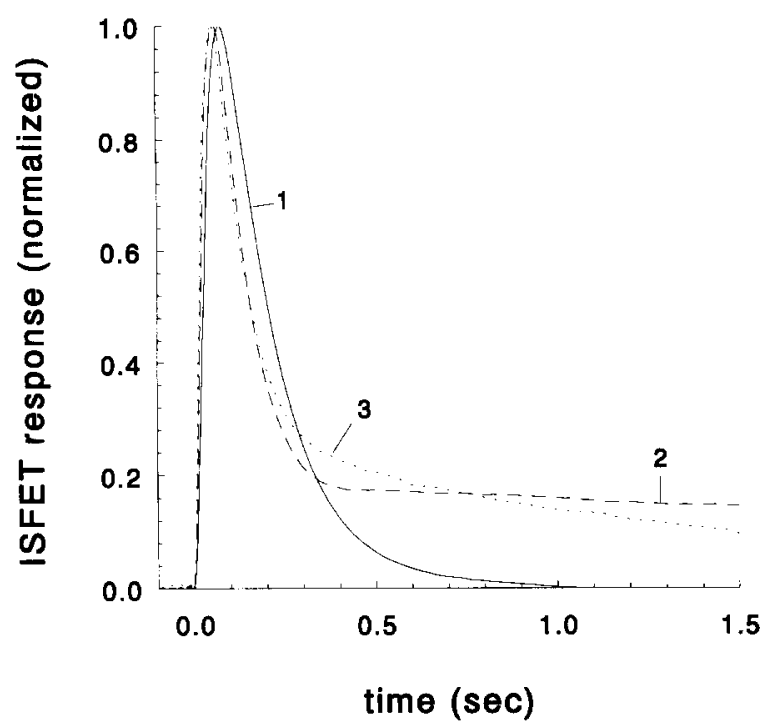

Fig. 8. The same ion-step responses as in Fig. 2 but here normalized to the amplitude. Curve 1 is the response of a bare ISFET, curve 2 of an ISFET with a protamine layer and curve 3 the response after incubation in a heparin solution.

the binding of heparin can still be described as a modification of the surface charge. If the previously mentioned membrane effects should contribute to the ion-step response, this would certainly influence the time constants of the ionstep response of the ISFET. It should be noted that curve 1 was recorded in solutions that were buffered with $0.2 \mathrm{mM}$ HEPES, of which the buffer capacity differs a little from the solutions buffered with $0 \cdot 1 \mathrm{mM}$ phosphate buffer used for recording curve 2 and 3 . Because the $\mathrm{pH}$ was not exactly the same in both ion-step solutions, curve 2 and 3 do not return to $0 \mathrm{mV}$ for $\mathrm{t} \rightarrow \infty$.

The amplitude of the ion-step response of an ISFET with a protamine layer is typically $9 \mathrm{mV}$ (Fig. 2, curve 2). If it is also assumed in this case that the response reaches $54 \%$ of its maximum, as described by eqn. 4 , a value of $17 \mathrm{mV}$ is obtained for $\Delta \Psi_{\max }$. Because the binding of the protamine results in a decrease in the absolute value of the surface potential $\Psi$, the value for the ratio $\mathrm{C}_{\mathrm{d} 11} / \mathrm{C}_{\mathrm{d} 12}$ is also changed (see Fig. 7). From eqn. 4 and Fig. 7, it can be calculated that the new ratio $\mathrm{C}_{\mathrm{dl1}} / \mathrm{C}_{\mathrm{d} 12}$ is 0.69 , which corresponds to a potential $\Psi_{1}$ (at $10 \mathrm{mM}$ ) of $-55 \mathrm{mV}$. This potential results in a surface charge of $-0.70 \times 10^{-6} \mathrm{C} / \mathrm{cm}^{2}$, or $4.4 \times 10^{12}$ negatively charged groups per $\mathrm{cm}^{2}$. With respect to a bare ISFET, the net surface charge is therefore decreased from 18.7 to $4.4 \times 10^{12}$ negatively charged groups per $\mathrm{cm}^{2}$. This means that the adsorbed protamine contributes to a positive charge density of $14.5 \times 10^{12}$ groups per $\mathrm{cm}^{2}$. According to the amino acid content of protamine (Cundall et al., 1982) a molecular charge of +20 can be assumed, which yields a coverage of $7 \times 10^{-3}$ molecules per $\mathrm{nm}^{2}$. This indicates that the protamine layer is not a dense layer, which indicates that the $\mathrm{Ta}_{2} \mathrm{O}_{5}$ surface is not completely covered with protamine.

The results of the measurements of heparin concentrations in PBS show that four different ISFETs behave in virtually the same way with respect to the heparin concentration in $\mathrm{PBS}$, as is shown in Table 1 (ISFET D at another $\mathrm{pH}$, using another heparin). The offsets fall within the accuracy of the measurement of $\Delta \mathrm{A}$ (mean value $\pm 0.4 \mathrm{mV}$ ) and the slopes are almost equal. According to the accuracy of $\Delta \mathrm{A}( \pm 0.4 \mathrm{mV})$, the accuracy in the measurement of the heparin concentration in PBS should be \pm 0.04 Units $/ \mathrm{ml}$ (using a mean slope of $11.1 \mathrm{mV}$.Unit ${ }^{-1} \cdot \mathrm{ml}^{-1}$ ). However, the curve fit does not include all data points within the accuracy as given by the error bars. A possible cause for this extra inaccuracy might be found in the procedure. During the two mins incubation time, the sample is not stirred; this makes the affinity reaction at the surface very sensitive for convection in the solution, to which no special attention was paid. Moreover, the incubation is manually timed, which introduces an estimated error of about 5 s $(4 \%)$.

The interaction of heparin with a protamine loaded surface has been described by a Langmuir adsorption model (Kim et al., 1992). As a function of time, Kim et al., found a rapid increase in the heparin concentration at the surface during the first 10 mins, followed by an asymptotic approach to a steady state value. It can therefore be concluded that during the 2 mins of incubation time used in our procedure, no equilibrium or steady state value can have been reached. Therefore, the binding to the ISFET cannot be described by a Langmuir adsorption model, as is also obvious from the linear relation between the heparin concentration and $\Delta \mathrm{A}$. More experiments will be needed to study the binding of heparin to the protamine treated ISFET as a function of time, which makes it possible to optimize the incubation time. The observation that the amount of bound heparin increases rapidly during the first minutes 
indicates that it is important to time the incubation period accurately.

The measurements in normal blood plasma show a significant offset that is probably caused by a non-specific interaction of plasma components with the ISFET surface. Because it has been reasoned that the coverage of protamine is not very dense, the non-specific interaction might include interaction with protamine molecules, as well as adsorption on "free" $\mathrm{Ta}_{2} \mathrm{O}_{5}$. This nonspecific interaction reduces the detection limit of heparin in plasma to about $0.25 \mathrm{Units} / \mathrm{ml}$. A more dense coverage of the surface with protamine, for instance by covalently coupling the protamine to the surface, might reduce the non-specific adsorption on the $\mathrm{Ta}_{2} \mathrm{O}_{5}$ surface and therefore reduce the offset and improve the detection limit. Another difference in comparison with the measurement of the heparin concentration in PBS is the slope of the linear curve fit corresponding to the relation between $\Delta \mathrm{A}$ and the heparin concentration, which is significantly smaller in the case of measurements in plasma. A possible explanation would be a smaller amount of heparin bound to the protamine during the incubation time of $2 \mathrm{mins}$ and/or an attempt by heparinbinding proteins, immobilized at the ISFET surface together with the heparin, to compensate the charge of the heparin molecules. It is known that protamine is able to bind all possible active heparin molecules, and protamine has successfully been used to separate all active heparin from plasma samples (Kim et al., 1992). The kinetics of heparin in plasma could be different because of binding to proteins, which might reduce the amount of bound heparin during the $2 \mathrm{~min}$. incubation time. Further research is necessary in order to understand the exact mechanism. This includes the observation that the slopes of the linear curve fit corresponding to the measurement in plasma are different for the three ISFETs, while the slopes of the linear fit of the measurements in PBS are almost alike.

The accuracy of the measured heparin concentration in plasma, according to the accuracy in $\Delta \mathrm{A}$, is 0.07 Units $/ \mathrm{ml}$ for ISFET $\mathrm{A}$, presented in Fig. 4 and 0.08 Units $/ \mathrm{ml}$ for ISFET D, presented in Fig. 5. In these cases an extra inaccuracy might also be caused by the procedure, as explained earlier.

Measurements of the heparin concentration in the patient plasma samples, as shown in Table 2 , show that in 9 out of 13 samples the added amount of 0.5 Units $/ \mathrm{ml}$ was correctly measured within the accuracy of the ISFET used $( \pm 0.08$ Units $/ \mathrm{ml}$ ). In these cases the calibration curve, which was recorded in normal plasma, could be used to determine the concentration in the individual plasma samples. The measured increase in the concentration in the four other samples was smaller than 0.5 Units $/ \mathrm{ml}$. Apparently the calibration curve obtained from measurements in normal plasma cannot be used in these cases. It is unclear which effects cause this difference; more specific experiments will be needed to investigate these effects.

From Fig. 6 it is obvious that there is no direct relation between the heparin concentration and the APTT. This finding is not surprising because the APTT is dependent on several other parameters (e.g. the concentration of AtIII and other clotting factors) that may vary from one patient to another. The therapeutic range for the APTT as used in the MST hospital has been defined as 70 to $120 \mathrm{sec}$ (normal values $25-35 \mathrm{sec}$ ). From Fig. 6 it can be seen that some of the plasma samples show clotting times within the therapeutic range whereas the heparin concentration appears to be $>1.0$ Units $/ \mathrm{ml}$. In another case the APTT clearly exceeds the therapeutic range (156 sec) whereas the heparin concentration appears to be only $0.85 \mathrm{Units} / \mathrm{ml}$. These results support the statement that the APTT is not a suitable assay with which to monitor heparin concentrations. Jaques et al., (1990) also determined absolute heparin concentrations in patient plasma samples and plotted the APTT as function of the heparin concentration resulting in a plot similar to Fig. 6 , in which no relation could be determined. The heparin concentration was determined by first separating the heparin from the plasma followed by microelectrophoresis to determine the absolute amount (Jaques et al., 1990). In this context, it would be interesting to compare the heparin concentration as measured with the heparin sensor system described in this paper, with the result of a specific chromogenic substrate test that uses additional AtIII and gives a result which is related to the concentration of active heparin molecules.

In this paper a heparin sensor system has been described that can determine absolute heparin concentrations in blood plasma between 0.25 and 2.0 Units $/ \mathrm{ml}$ with an accuracy of \pm 0.08 Units/ $\mathrm{ml}$. A detection limit of 0.25 Units $/ \mathrm{ml}$ in blood plasma corresponds to about $13.3 \times 10^{-8} \mathrm{~mol} / \mathrm{l}$ 
assuming an average molecular weight of 15000 and a value of $8 \mu \mathrm{g}$ per unit. ${ }^{*}$ With respect to the heparin sensor that uses a porous membrane on top of the ISFET, as described earlier (Van Kerkhof et al., 1993), the non-specific interactions with other plasma components is significantly reduced. The incubation time is also reduced from 15 to 2 mins. More experiments are needed to study the behaviour of the sensor in individual plasma samples. The specifications of the sensor system can still be optimized by establishing a higher degree of coverage of the $\mathrm{Ta}_{2} \mathrm{O}_{5}$ surface with protamine, which might reduce the nonspecific interactions of other plasma components and increase the sensitivity to heparin concentrations. The procedure can be optimized by a more accurate timing of the incubation time and by stirring the sample during incubation.

\section{ACKNOWLEDGEMENTS}

The authors thank Johan Bomer for the realization and encapsulation of the ISFETs and for technical assistance with the measurement setup. Kees Schenkenveld from the laboratory of the "Medisch Spectrum Twente" Hospital is gratefully acknowledged for providing all plasma samples and the MST heparin. This research project is financially supported by the Netherlands Organization of Applied Scientific Research TNO.

\section{REFERENCES}

Abildgaard, U. (1989). Monitoring heparin treatment. In: Heparin, Chemical \& Biological Properties, Clinical Applications, (D.A. Lane \& U. Lindahl, eds.), Edward Arnold, London.

Bard, A.J. \& Faulkner, L.R. (1980). Electrochemical Methods, Fundamentals \& Applications, John Wiley \& Sons, New York.

Barrowcliffe, T.W. (1989). Heparin assays and standardization. In: Heparin, Chemical \& Biological Properties, Clinical Applications, (D.A. Lane \& U. Lindahl, eds.), Edward Arnold, London.

\footnotetext{
* In our previous paper (van Kerkhof et al., 1993) we mentioned that $0 \cdot 1$ Units $/ \mathrm{ml}$ corresponds to $5 \times 10^{-11} \mathrm{~mol} / \mathrm{l}$ using a molecular weight of 15000 and a value of $8 \mu \mathrm{g} /$ Unit. However, this value is not correct and should be $5 \times 10^{-8} \mathrm{~mol} / \mathrm{l}$.
}

Bärtl, K., Dorsch, E., Lill, H. \& Ziegenhorn, J. (1980). Determination of the biological activity of heparin by use of a chromogenic substrate. Thromb. \& Haemost., 42, 1446.

Björk, I., Olson, S.T. \& Shore, J.D. (1989). Molecular mechanisms of the accelerating effect of heparin on the reactions between antithrombin and clotting proteinases. In: Heparin, Chemical \& Biological Properties, Clinical Applications, (D.A. Lane \& U. Lindahl, eds.), Edward Arnold, London.

Björnsson, T.D. \& Wolfram, K.M. (1982). Intersubject variability in the anticoagulant response to heparin in vitro. Euro. J. of Clin. Pharm., 21, 491.

Casu, B. (1989). Structure of heparin and heparin fragments. Ann. N.Y. Acad. Sci., 556, 1-17.

Cundall, R.B., Jones, G.R. \& Murray, D. (1982). Polyelectrolyte complexes, 3. The interaction between heparin and protamine. Makromol. Chem., 183, 849-861.

van Hal, R.E.G., Eijkel, J.C.T. \& Bergveld, P. (1994). The $\mathrm{pH}$ sensitivity of ISFETs described in terms of buffer capacity and double layer capacitance. Tech. Digest 5th Intl. meeting Chem. Sensors, Rome, 11-14 July, 1994.

Hirsch, J. (1986). Mechanism of action and monitoring of anticoagulants. Seminars in Thromb. \& Haemost., 12, 1.

Holm, H.A., Kalvenes, S. \& Abildgaard, U. (1985). Changes in plasma antithrombin (heparin cofactor activity) during intravenous heparin therapy: observations in 198 patients with deep venous thrombosis. Scand. J. Haemost., 35, 564-569.

Holm, H.A., Abildgaard, U., Larsen, M.L. \& Kalvenes, S. (1987). Monitoring of heparin therapy: should heparin assays also reflect the patient's antithrombin concentration?. Thromb. Res., 46, 669-675.

Höök, M., Björk, I., Hopwood, J.J. \& Lindahl, U. (1976). Anticoagulant activity of heparin: separation of high-activity and low-activity heparin species by affinity chromatography on immobilized antithrombin. Fed. Eur. Biochem. Soc. Lett., 66, 90-93.

Jaques, L.B., Wice, S.M. \& Hiebert, L.M. (1990). Determination of absolute amounts of heparin and of dextran sulphate in plasma in microgram quantities. J. Lab. Clin. Med., 115, 422-432.

van Kerkhof, J.C., Bergveld, P. \& Schasfoort, R.B.M. (1993). Development of an ISFET based heparin sensor using the ion-step measuring method. Biosensors \& Bioelectr., 8, 463-472.

van Kerkhof, J.C., Eijkel, J.C.T. \& Bergveld, P. (1994a). ISFET responses on a stepwise change in electrolyte concentration at constant $\mathrm{pH}$. Sensors \& Actuators B, 18, 56-59.

van Kerkhof, J.C., Bergveld, P. \& Schasfoort, R.B.M. (1994b). A heparin sensor based on a modified 
$\mathrm{Ta}_{2} \mathrm{O}_{5}$-ISFET and the ion-step measuring method. Proc. of Dutch Conf. Sensor Tech. 1994, Univ. of Twente, Enschede, The Netherlands, Feb. 17-18, 1994, (P.V. Lambeck, ed.) ISBN 90-73461-06-5, pp. 281-285.

Kim, J., Yang, A.J. \& Yang, V.C. (1992). Protamine immobilization and heparin adsorption on the protamine-bound cellulose fibre membrane. Biotech. \& Bioengin., 39, 450-456.

Klein, M.D., Drongowski, R.A., Linhardt, R.J. \& Langer, R.S. (1982). A colorimetric assay for chemical heparin in plasma. Anal. Biochem., 124, $59-64$.

Levy, S.W. \& Jaques, L.B. (1978). Appearance of heparin anti-thrombin-active chains in vivo after injection of commercial heparin and in anaphylaxis. Thromb. Res., 13, 429-441.

Linhardt, R.J. (1991). Heparin: an important drug enters its seventh decade. Chem. \& Ind., Jan., 45-50.

Ma, S.-C, Yang, V.C. \& Meyerhoff, M.E. (1992). Heparin-responsive electrochemical sensor: a preliminary study. Anal. Chem., 64, 694-697.

Ma, S.-C., Yang, V.C., Fu, B. \& Meyerhoff, M.E. (1993). Electrochemical sensor for heparin: further characterization and bioanalytical applications. Anal. Chem., 65, 2078-2084.

Nader, H.B., Dietrich, C.P. (1989). Natural occurrence and possible biological role of heparin. In: Heparin, Chemical \& Biological Properties, Clinical Applications, (D.A. Lane \& U. Lindahl, eds.), Edward Arnold, London.
Petitou, M. (1989). Chemical synthesis of heparin. In: Heparin, Chemical \& Biological Properties, Clinical Applications, (D.A. Lane \& U. Lindahl, eds.), Edward Arnold, London.

van Putten, J.J., van de Ruit, M. Beunis, M. \& Hemker, H.C. (1982). Interindividual variation in relationships between plasma heparin concentration and the results of five heparin assays. Clinica Chim. Acta, 122, 261.

Rosenberg, R.D. (1987). The heparin-antithrombin system: a natural anticoagulant mechanism, In: Haemostasis \& Thrombosis, Basic Principles and Clinical Practice. (R.W. Colman, J. Hirsch, V.J. Marder \& E.W. Salzman, eds.), J.B. Lippincott Co., Philadelphia.

Schasfoort, R.B.M., Bergveld, P., Kooyman, R.P.H. \& Greve, J. (1990). A new approach to ImmunoFET operation. Biosensors \& Bioelect., 5, 103-124.

Teien, N.A. \& Lie, M. (1975). Heparin assay in plasma. A comparison of five clotting methods. Thromb. Res., 7, 777.

Teien, N.A. \& Lie, M. (1977). Evaluation of an amidolytic heparin assay method: Increased sensitivity by adding purified antithrombin III. Thromb. Res., 10, 399.

Yang, V.C., Ma, S.-C., Liu, D., Brown, R.D. \& Meyerhoff, M.E. (1993). A novel electrochemical heparin sensor. ASAIO J., 39, 195-201. 